\title{
In-body path loss models for implants in heterogeneous human tissues using implantable slot dipole conformal flexible antennas
}

\author{
Divya Kurup ${ }^{1 *}$, Maria Scarpello², Günter Vermeeren ${ }^{1}$, Wout Joseph ${ }^{1}$, Kristof Dhaenens ${ }^{3}$, Fabrice Axisa ${ }^{3}$, \\ Luc Martens ${ }^{1}$, Dries Vande Ginste ${ }^{2}$, Hendrik Rogier ${ }^{2}$ and Jan Vanfleteren ${ }^{3}$
}

\begin{abstract}
A wireless body area network (WBAN) consists of a wireless network with devices placed close to, attached on, or implanted into the human body. Wireless communication within a human body experiences loss in the form of attenuation and absorption. A path loss model is necessary to account for these losses. In this article, path loss is studied in the heterogeneous anatomical model of a 6-year male child from the Virtual Family using an implantable slot dipole conformal flexible antenna and an in-body path loss model is proposed at $2.45 \mathrm{GHz}$ with application to implants in a human body. The model is based on 3D electromagnetic simulations and is compared to models in a homogeneous muscle tissue medium.
\end{abstract}

\section{Introduction}

A wireless body area network (WBAN) is a network, consisting of nodes that communicate wirelessly and are located on or in the body of a person. These nodes form a network that extends over the body of the person. Depending on the implementation, the nodes consist of sensors and actuators, placed in a star or multihop topology [1].

Applications of WBANs include medicine, sports, military, and multimedia, which make use of the freedom of movement provided by the WBAN. As WBAN facilitates unconstrained movement amongst users, it has brought a revolutionary change in patient monitoring and health care facilities. Active implants placed within the human body lead to better and faster diagnosis, thus improving the patient's quality of life. Implantable devices are increasingly proving their importance for biomedical applications. The use of active implants allows vital medical data to be collected over a longer period in the natural environment of the patient, allowing for a more accurate and sometimes even faster diagnosis. Active implants such as pacemakers and

\footnotetext{
* Correspondence: divya.kurup@intec.ugent.be

'Department of Information Technology, WiCa, Ghent University/IBBT,

Gaston Crommenlaan 8 Box 201, 9050 Ghent, Belgium

Full list of author information is available at the end of the article
}

implantable cardioverter defibrillators (ICDs) need to relay information to other devices for control or monitoring [2]. Thus a proper and efficient modeling of the channel is required to transfer data between implants and other devices. Moreover, the human body is a lossy medium which attenuates the waves propagating from the transmitter $(\mathrm{Tx})$ considerably before they reach the receiver $(\mathrm{Rx})$. Thus, to design an optimal communication link between nodes placed within or on the human body a proper and efficient path loss (PL) model is required.

To our knowledge very limited literature exists on propagation loss within the human body [2-6]. In [3] initial results of an in-body propagation model in saline water is presented. Inaccuracies lead to maximum deviations of $9 \mathrm{~dB}$ between the measurements and simulations. Also only a homogeneous medium is studied and there are no models available for heterogeneous medium. [3] considers a non-insulated hertzian dipole, hence the PL model can only be applied to very small dipole antennas. [4] provides various scenarios for channel modeling but does not provide a model for path loss. [5] discusses a link budget for an implanted cavity slot antenna at $2.45 \mathrm{GHz}$. However, no model for a heterogeneous medium is suggested that can be used for path loss simulation. [2] suggests a PL model for in-

\section{SpringerOpen ${ }^{\circ}$}

(c) 2011 Kurup et al; licensee Springer. This is an Open Access article distributed under the terms of the Creative Commons Attribution License (http://creativecommons.org/licenses/by/2.0), which permits unrestricted use, distribution, and reproduction in any medium, provided the original work is properly cited. 
body wireless implants. However, it does not make use of biocompatible implantable antennas. [6] suggests a PL model for in-body wireless implants by making use of insulated dipole antennas in a homogeneous medium.

The goal of this article is to develop an empirical PL model for a heterogeneous medium, using implantable antennas, that describes the relationships between the $\mathrm{PL}$, the distance between the antennas, and the power attenuation. Since it is difficult to carry out measurements in the human body, implantable antennas are designed by taking the dielectric properties of human muscle tissue into consideration.

Simulations are performed at $2.45 \mathrm{GHz}$ in the license free industrial, scientific, and medical (ISM) band. This frequency band is chosen since there are no licensing issues in this band and the higher frequency allows the use of a smaller antenna. Moreover, $2.45 \mathrm{GHz}$ allows higher bitrates due to the larger bandwidth [7]. After carrying out the simulations in human muscle tissue, simulations are carried out in a heterogeneous medium for various scenarios using an enhanced anatomical model of a 6-year-old male child from the Virtual Family (Christ, in preparation). We use a child model because in children with evidence of internal bleeding and abdominal pain, correct diagnosis is a challenge and capsule endoscopy can be used for the diagnosis of such ailments. Capsule endoscopy has been accepted in adults by many gastroenterologists, however its usage in children has lagged due to the belief by pediatricians that the pills are too large to be swallowed by children $[8,9]$. However, reports do suggest that children as young as two and a half years old are successfully undergoing capsule endoscopy, and most of the studies suggest that majority of pediatric patients can swallow the pill $[10,11]$.

The PL model developed in this article focuses on deep tissue implants, such as endoscopy capsules. In such applications the implants are placed deep inside the body, which we have selected up to a distance of 8 $\mathrm{cm}$. A PL model will help in understanding the influence of the dielectric properties of the surrounding tissues and the power attenuation of such implants. As it is difficult for the manufacturers to test their system on actual humans, the proposed model can be used by them to evaluate the performance of in-body WBAN systems using well specified setups and to carry out link budget calculations.

The outline of this article is as follows. The setup and configuration of the simulations in the homogeneous muscle tissue medium and the heterogeneous human model are discussed in Sects. II and III, respectively. Section IV discusses the results including the reflection coefficient and the path loss of the implanted antennas in human muscle tissue medium and the heterogeneous model. Section V presents the conclusions.

\section{Homogeneous Tissue: Human Muscle Tissue \\ A. Setup and configuration}

We first investigate wave propagation at $2.45 \mathrm{GHz}$ in human muscle tissue (relative permittivity $\varepsilon_{\mathrm{r}}=50.8$ and conductivity $\sigma=2.01 \mathrm{~S} / \mathrm{m}$ [12]), using simulations for implantable antennas. These implantable antennas operating in the $2.45 \mathrm{GHz}$ ISM band are designed based on recommendations set by the European Radiocommunications Committee (ERC) for ultra-low-power active medical implants [13]. We consider the implantable antenna as a short range device (SRD) working in the ISM band because SRD is intended to cover the radio transmitters which provide either unidirectional or bidirectional communication and have low capability of causing interference to other radio equipment. SRDs use either integral, dedicated or external antennas and all modes of modulation can be permitted subject to relevant standards. The antennas are flexible folded slot dipole antennas embedded in biocompatible polydimethylsiloxane (PDMS) to make it suitable for implantation [7]. The flexible property of the antenna makes it more convenient to be placed in different parts of the body instead of placing a rigid structure. The antenna is manufactured using flexible electronic technology: the metallization resides on a flexible polyimide substrate with a thickness of $25 \mu \mathrm{m}$, a relative permittivity of $\varepsilon_{\mathrm{r}}=$ 3.5 , and a loss tangent of $\tan \delta=0.003$. Two PDMS layers are used as substrate and superstrate, each with a thickness of $2.5 \mathrm{~mm}$. The dielectric properties of the PDMS were characterized at $2.45 \mathrm{GHz}$, to be $\varepsilon_{\mathrm{r}}=2.2$ and $\tan \delta=0.013$. The top view of the coplanar waveguide (CPW) fed antenna is shown in Figure 1 and its dimensions are presented in Table 1 . The antenna length $(L=25.9 \mathrm{~mm})$ may seem to be slightly large for an immediate in human body, however, this is the starting point to proceed with further development. It is also feasible to shorten the CPW, matched at $50 \mathrm{Ohm}$, whose length is now $18 \mathrm{~mm}$ and the saved space can then be used for the insertion of an IC package.

\section{1) Simulation}

Simulations are performed using a 3D electromagnetic solver SEMCAD-X (SPEAG, Switzerland), a finite-difference time-domain (FDTD) program. SEMCAD-X enables non-uniform gridding. The maximum grid step in the muscle tissue medium is $1 \mathrm{~mm}$ at $2.45 \mathrm{GHz}$. The simulations are carried out using the implantable antennas up to a distance of $8 \mathrm{~cm}$. The muscle tissue is modeled by using a cube (dimensions $150 \times 150 \times 280$ $\mathrm{mm}^{3}$ ) with the dielectric properties of human muscle tissue. The implantable antennas are aligned for 


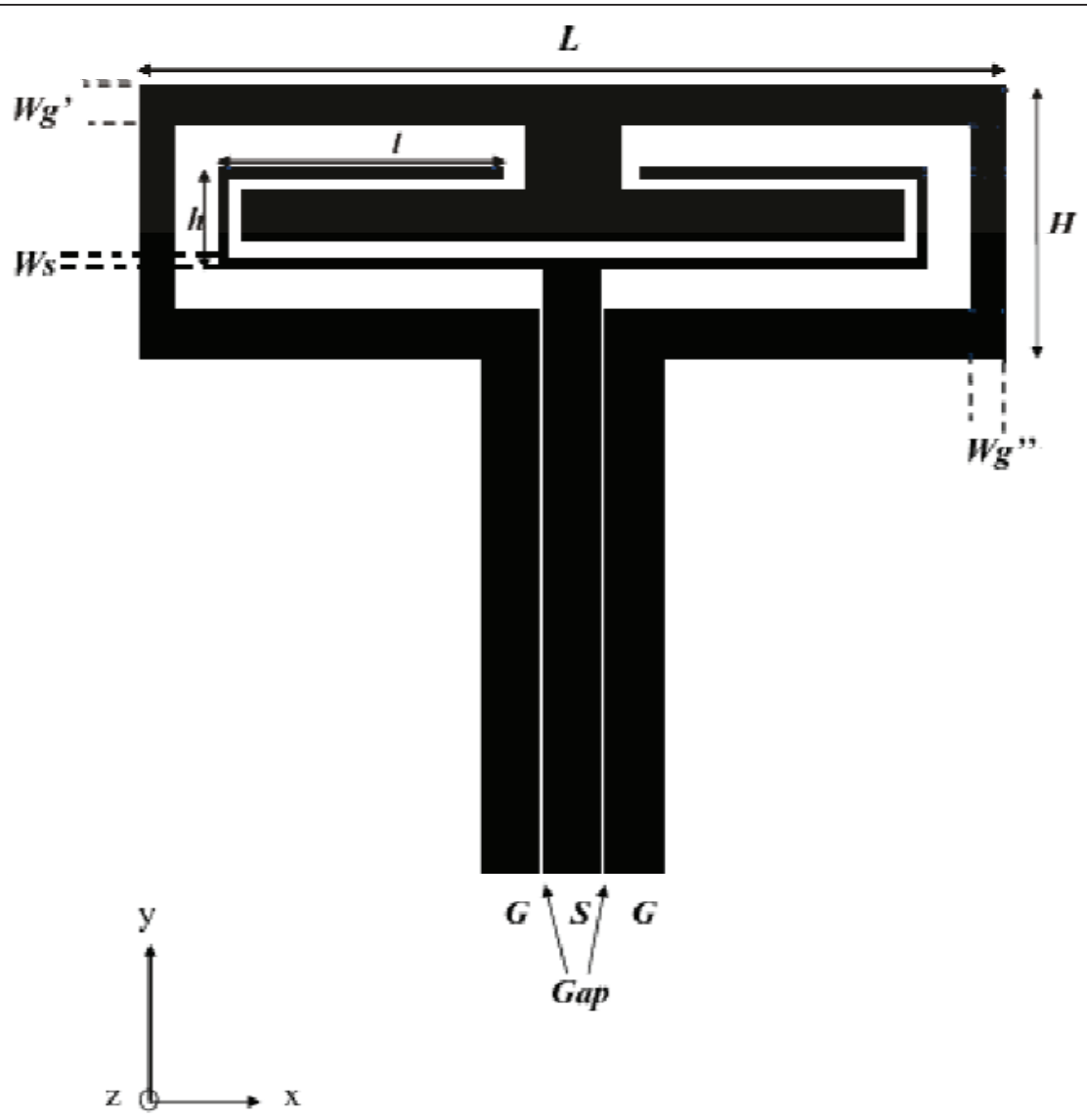

Figure 1 Top view of the coplanar waveguide-fed antenna.

maximum power transfer and the source used is a voltage source.

\section{Heterogeneous Medium}

\section{A. Setup and configuration}

We also simulate to investigate wave propagation at 2.45 $\mathrm{GHz}$ in a heterogeneous medium using the real implantable antenna proposed in Figure 1. The heterogeneous medium is an enhanced anatomical model of a 6-yearold male child from Virtual Family (Figure 2) (Christ, in preparation). The model is based on magnetic resonance images (MRI) of healthy volunteers. The male child model (virtual family boy, VFB) has a height of $1.17 \mathrm{~m}$ and a weight of $19.5 \mathrm{~kg}$. The model consists of 81 different tissues. The dielectric properties of the body tissues have been taken from the Gabriel database [14]. Simulations to determine PL are carried out using the FDTD solver in SEMCAD-X (SPEAG, Switzerland). The

Table 1 Size of the folded slot dipole antenna

\begin{tabular}{|c|c|c|c|c|c|c|c|c|c|c|c|}
\hline Unit & $\mathrm{H}$ & $L$ & $h$ & $I$ & $\mathrm{Wg}^{\prime}$ & $\mathrm{Wg}^{\prime \prime}$ & Wg'"' & Ws & G & $S$ & Gap G-S \\
\hline$(\mathrm{mm})$ & 8.5 & 25.9 & 3.2 & 8.3 & 1.2 & 1.5 & 1.0 & 0.3 & 1.8 & 1.7 & 0.1 \\
\hline
\end{tabular}

implantable antennas are placed in the trunk of the male child model to determine PL from a distance of 1 $\mathrm{cm}$ up to $8 \mathrm{~cm}$ for applications such as an endoscopy capsule. The maximum step in grid setting is $1 \mathrm{~mm}$. The padding, which is the spacing added to the grid from the bounding box of the model to the grid boundary, is negative so that the grid covers the part of the human body entirely. The absorbing boundary condition used is very high mode with a very high strength thickness, where a minimum level of absorption at the outer boundary is $99.99 \%$ [15]. A transient excitation of 30 periods is set to ensure that a steady state is reached. Since the simulation using the whole body of the male child model consumes a lot of time, the simulation domain is reduced to just cover the trunk of the male child model, however, validation for some cases has been done with the full body of the VFB. Simulations are carried out for various scenarios taking the path of an endoscopy capsule into consideration:

- Scenario I- Esophagus: For each scenario, the Tx and the $\mathrm{Rx}$ are placed at three different positions. The first position is at location 1 as shown in the 


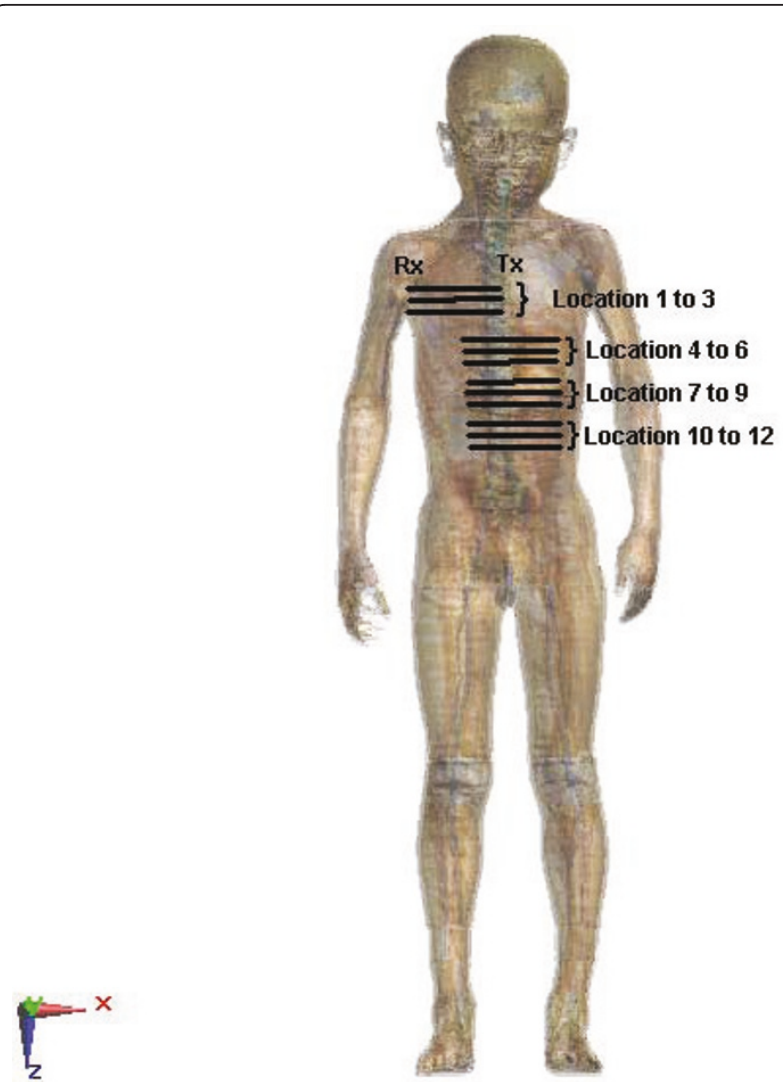

Figure 2 Locations of Tx and Rx for various scenarios in the VFB.

Figure 2. Here the Tx antenna is placed in the esophagus $\left(\varepsilon_{\mathrm{r}}=62.15\right.$ and $\left.\sigma=2.2 \mathrm{~S} / \mathrm{m}\right)$ of the VFB and the receiving antenna is placed at a separation of $1 \mathrm{~cm}$ from the Tx up to $5 \mathrm{~cm}$ in steps of $5 \mathrm{~mm}$ as shown in the Figure 2. The $\mathrm{Rx}$ antenna traverses through the lungs $\left(\varepsilon_{\mathrm{r}}=34.42\right.$ and $\left.\sigma=1.24 \mathrm{~S} / \mathrm{m}\right)$ in this position. Position 2 in this scenario is such that the $\mathrm{Tx}$ and $\mathrm{Rx}$ antennas are placed $1 \mathrm{~cm}$ below location 1 and this is indicated as location 2 in the Figure 2. In position 3 the $\mathrm{Tx}$ and $\mathrm{Rx}$ antennas are placed $2 \mathrm{~cm}$ below location 1 and are indicated as location 3 in the Figure 2. At all these positions the Rx antenna again traverses through the lungs.

- Scenario II-Stomach: Here, the implantable antenna is placed such that the Tx lies in the stomach and the Rx moves from $1 \mathrm{~cm}$ to $2 \mathrm{~cm}$ through the stomach lumen $\left(\varepsilon_{\mathrm{r}}=52.72\right.$ and $\left.\sigma=1.74 \mathrm{~S} / \mathrm{m}\right)$, which is enclosed by stomach $\left(\varepsilon_{\mathrm{r}}=62.16\right.$ and $\sigma=$ $2.21 \mathrm{~S} / \mathrm{m})$, and then moves partially into the liver $\left(\varepsilon_{\mathrm{r}}\right.$ $=54.81$ and $\sigma=2.25 \mathrm{~S} / \mathrm{m}$ ) starting from $3 \mathrm{~cm}$ and and then entirely up to $8 \mathrm{~cm}$ as shown in location 4 in Figure 2. Position 2 and position 3 in this scenario are such that the $\mathrm{Tx}$ and $\mathrm{Rx}$ antennas are placed 1 $\mathrm{cm}$ and $2 \mathrm{~cm}$ below location 4 indicated as location 5 and location 6 in the Figure 2.

- Scenario III-Small intestine: In the first position of this scenario the Tx antenna is placed in the small intestine $\left(\varepsilon_{\mathrm{r}}=54.42\right.$ and $\sigma=3.17 \mathrm{~S} / \mathrm{m}$ at $\left.2.45 \mathrm{GHz}\right)$ of the VFB as shown in Figure 2 at location 7. The $\mathrm{Rx}$ antenna is placed starting from $1 \mathrm{~cm}$ up to a separation of $8 \mathrm{~cm}$ from the $\mathrm{Tx}$ antenna. In this position the $\mathrm{Rx}$ antenna traverses through various tissues such as the kidney $\left(\varepsilon_{\mathrm{r}}=52.74\right.$ and $\sigma=2.43$ $\mathrm{S} / \mathrm{m})$, gall bladder $\left(\varepsilon_{\mathrm{r}}=68.36\right.$ and $\left.\sigma=2.8 \mathrm{~S} / \mathrm{m}\right)$, liver $\left(\varepsilon_{\mathrm{r}}=54.81\right.$ and $\left.\sigma=2.25 \mathrm{~S} / \mathrm{m}\right)$, and also the artery $\left(\varepsilon_{\mathrm{r}}\right.$ $=58.26$ and $\sigma=2.54 \mathrm{~S} / \mathrm{m}$ ). Position 2 and position 3 in this scenario are such that the Tx and Rx antennas are placed $1 \mathrm{~cm}$ and $2 \mathrm{~cm}$ below location 7 indicated as location 8 and location 9 in Figure 2.

- Scenario IV-Large intestine: The Tx antenna is placed in the large intestine $\left(\varepsilon_{\mathrm{r}}=53.87\right.$ and $\sigma=2.03$ $\mathrm{S} / \mathrm{m}$ at $2.45 \mathrm{GHz}$ ) of the VFB as shown in Figure 2 at the location 10. Here the $\mathrm{Rx}$ antenna traverses through the large intestine and also through fat $\left(\varepsilon_{\mathrm{r}}=\right.$ 5.28 and $\sigma=0.105 \mathrm{~S} / \mathrm{m}$ ). Position 2 and position 3 in this scenario are such that the $\mathrm{Tx}$ and $\mathrm{Rx}$ antennas are placed $1 \mathrm{~cm}$ and $2 \mathrm{~cm}$ below the location 10 indicated as location 11 and location 12 in Figure 2. In total, 162 simulations are carried out in the heterogeneous VFB for the various scenarios.

\section{Results}

A. Return loss for the implantable antenna: muscle tissue and heterogeneous medium

The simulated reflection loss of the Tx implantable antenna in the homogeneous muscle tissue medium, esophagus (scenario I), stomach (scenario II), small intestine (scenario III), and the large intestine (scenario IV) of the VFB as a function of frequency is shown in Figure 3. At $2.45 \mathrm{GHz}$ the antenna has an $\left|S_{11}\right|$ of $-29.21,-38,-29.7,-26.6,-31 \mathrm{~dB}$ in the homogeneous muscle tissue, the esophagus, the stomach, the small intestine and the large intestine of the VFB, respectively. As the antenna is developed by only taking the dielectric properties of the muscle tissue medium [7] into account, variations in effective permittivity (insulation and the medium) and wavelength in different tissues cause the variation in $\left|S_{11}\right|$. In particular, a shift of the resonance frequency of the antenna can be observed when placed in different tissues, still the values of the $\left|S_{11}\right|$ in all the scenarios are below $-10 \mathrm{~dB}$ in the complete ISM band from 2.40 to $2.48 \mathrm{GHz}$ and thus the antenna is suitable for in-body propagation with an ohmic loss of $2.5 \%$. The input impedance of the implantable antenna in the homogeneous muscle tissue at $2.45 \mathrm{GHz}$ is $Z(\Omega)=49.9$ - j3.4 whereas the input impedance of the implantable 


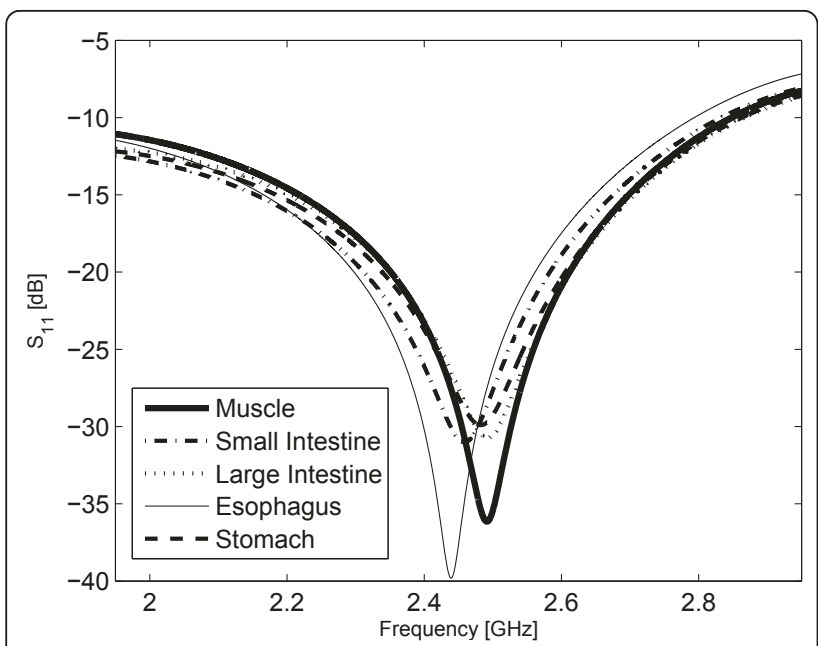

Figure 3 Reflection loss of the implantable antenna in homogeneous tissue and heterogeneous VFB.

antenna in the stomach, small intestine, large intestine, and esophagus of the VFB is equal to $53.79+j 8.75$, $51.63-j 3.9,52.40-j 1.7$, and $49.37+j 1.14$, respectively, which is about $50 \Omega$, as desired.

\section{B. Path loss}

PL is defined as the ratio of input power at port $1\left(P_{\text {in }}\right)$ to power received at port $2\left(P_{\text {rec }}\right)$ in a two-port setup. $\mathrm{PL}$ in terms of transmission coefficient is defined as $1 / /$ $\left.S_{21}\right|^{2}$ with respect to $50 \Omega$ when the generator at the Tx has an output impedance of $50 \Omega$ and the Rx is terminated with $50 \Omega$. This allows us to regard the setup as a two-port circuit for which we determine $\left|S_{21}\right|_{\mathrm{dB}}$ with reference impedances of $50 \Omega$ at both ports:

$$
\left.P L\right|_{\mathrm{dB}}=\left(P_{\text {in }} / P_{\text {rec }}\right)=-10 \log _{10}\left|S_{21}\right|^{2}=-\left|S_{21}\right|_{\mathrm{dB}},
$$

\section{1) $P L$ in human muscle tissue and heterogeneous VFB}

Figure 4 compares the simulated PL in human muscle tissue and the 162 simulations for the heterogeneous VFB as a function of distance $d$ for the implanted antenna. Figure 4 shows that the PL in the heterogeneous model which involves tissues with lower conductivities is lower than the PL in muscle tissue as in scenario I (esophagus) and scenario II (stomach). PL is seen to be larger in scenario III (small intestine) and scenario IV (large intestine) which involves tissues with higher conductivity as compared to the conductivity of the homogeneous muscle tissue. The maximum PL at 8 $\mathrm{cm}$ is obtained for the small intestine and is $75.8 \mathrm{~dB}$. In the homogeneous muscle tissue the slope of the PL remains constant, however in the heterogeneous scenarios the slope of the PL changes as the antenna moves from one tissue to another due to differences in the

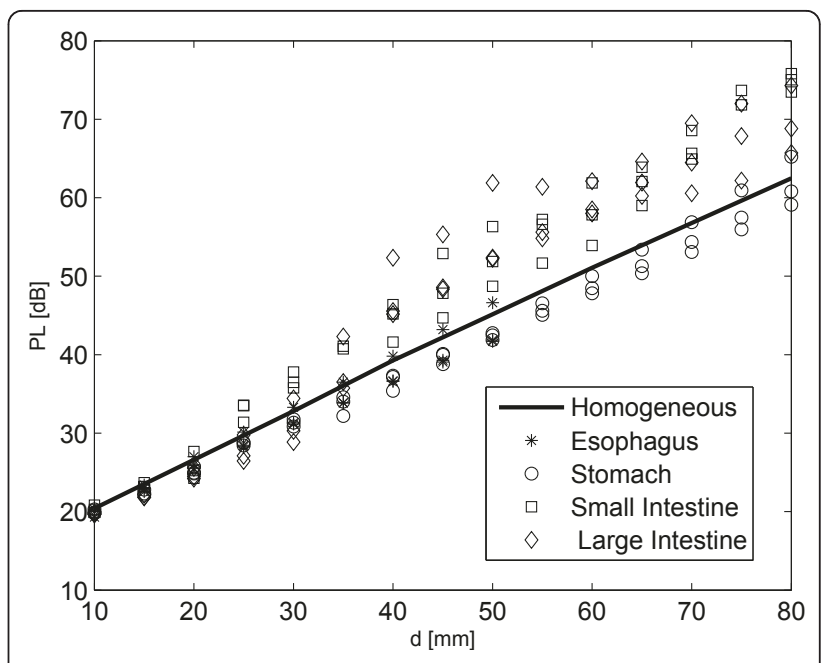

Figure 4 Path loss of the implantable antenna in the homogeneous medium and in heterogeneous VFB.

dielectric properties of the tissues through which the $\mathrm{Rx}$ antenna traverses. For example, in Figure 4, a change in the slope can be observed in the large intestine at a distance of $4 \mathrm{~cm}$ and $5 \mathrm{~cm}$ because the large intestine is thin and hence the Rx moves out into a region occupied by fat.

\section{PL model}

\section{1) Homogeneous human muscle tissue}

In this section the simulated results are used to develop a PL model as a function of distance in human muscle tissue at $2.45 \mathrm{GHz}$. The simulated results and the fitted model in human muscle tissue are shown in Figure 5. The PL is modeled as follows [6]:

$$
\left.P L\right|_{\mathrm{dB}}=\left(10 \log _{10} e^{2}\right) \alpha_{1} d+\left.C_{1}\right|_{\mathrm{dB}}
$$

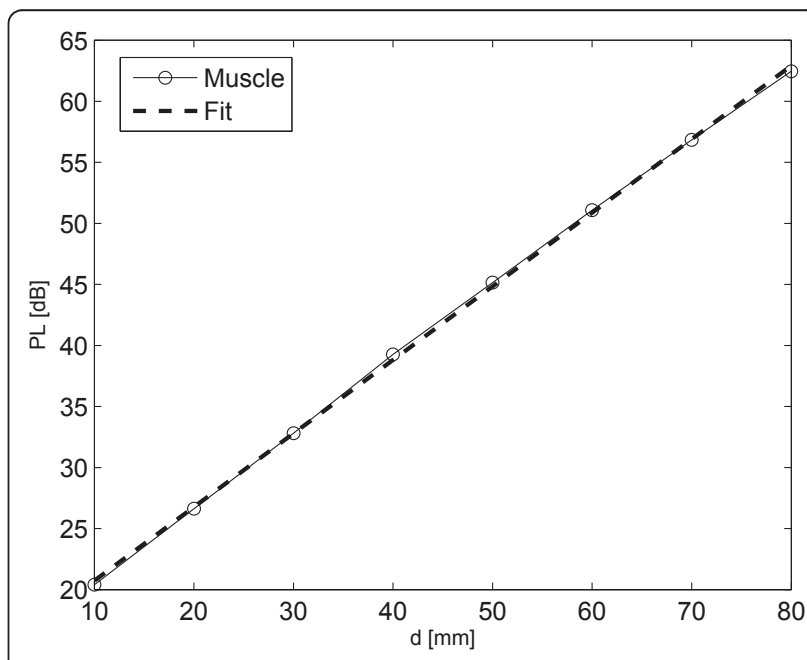

Figure $5 \mathrm{PL}$ in homogeneous muscle tissue and fitted model. 
where the parameter $\alpha_{1}$ is the attenuation constant $\left[\frac{1}{\mathrm{~cm}}\right],\left.C_{1}\right|_{\mathrm{dB}}$ is a constant and their values are listed in Table 2. $10 \log _{10} e^{2}$ equals $8.68 \mathrm{~dB}$ and shows the exponential behavior of the PL. The power decays exponentially with respect to distance in a lossy medium similar to the behavior of plane waves in lossy medium [16]. Since the fields exhibit an exponential decay in the medium, the trend of the PL in Figure 5 shows an exponential behavior in accordance to the linear regression equation (2).

\section{2) Heterogeneous model for esophagus-scenario I}

In this section the simulated results are used to develop a PL model as a function of distance for the Tx placed at the esophagus of the VFB at $2.45 \mathrm{GHz}$. The PL and the fitted model are shown in Figure 6. In this scenario the $\mathrm{Rx}$ antenna moves completely into the lungs at a separation of $2 \mathrm{~cm}$ from the Tx antenna. Up to $2 \mathrm{~cm}$ from Tx antenna a part of the Rx antenna moves in the heart muscle $\left(\varepsilon_{\mathrm{r}}=54.81\right.$ and $\left.\sigma=2.25 \mathrm{~S} / \mathrm{m}\right)$. Thus, a change of slope is observed at $2 \mathrm{~cm}$ where the antenna makes a transition from one tissue to another. This same behavior is noticed in all the scenarios for heterogeneous media when the antenna makes a transition from one tissue to another and can be observed very well when there is a huge difference in the dielectric properties. The PL is modeled as follows:

$$
\left.\mathrm{PL}\right|_{\mathrm{dB}}=\left(10 \log _{10} e^{2}\right) \alpha_{2} d+\left.C_{2}\right|_{\mathrm{dB}}+\left.\chi_{2}\right|_{\mathrm{dB}},
$$

where the parameter $\alpha_{2}$ is the effective attenuation constant $\left[\frac{1}{\mathrm{~cm}}\right],\left.C_{2}\right|_{\mathrm{dB}}$ is a constant and their values are listed in Table 2. The effective attenuation constant is the attenuation constant for all the tissues through which the Rx antenna traverses through in each scenario. The PL model is a linear regression model thus consisting of a deterministic part which is a function of distance and the random error term, $\left.\left.\chi_{2}\right|_{\mathrm{dB}} \cdot \chi_{2}\right|_{\mathrm{dB}}$ follows a zero mean normal distribution with a standard deviation (SD), of $1.31 \mathrm{~dB}$. Figure 7 shows the Q-Q plot of the empirical quantiles of the error between the PL model and the simulated PL results on the vertical axis to a theoretical standard normal distribution on the horizontal axis in the esophagus of the VFB. A Q-Q plot is a probability plot which compares two probability

Table 2 Parameter values and SD of the fitted models for $\mathrm{PL}_{\mathrm{dB}}$ in human muscle tissue and the heterogeneous

\begin{tabular}{ccccc}
\hline No. & Scenario & $\boldsymbol{\alpha}_{\mathbf{i}}\left(\frac{\mathbf{1}}{\mathbf{c m}}\right)$ & $\boldsymbol{C}_{\boldsymbol{i}}(\mathbf{d B})$ & $\mathbf{S D}(\mathbf{d B})$ \\
\hline I & Homogeneous muscle tissue & 0.69 & 14.71 & - \\
\hline II & VFB Esophagus & 0.67 & 14.24 & 1.31 \\
\hline III & VFB Stomach & 0.68 & 13.40 & 1.22 \\
\hline IV & VFB Small Intestine & 0.89 & 12.36 & 2.25 \\
\hline V & VFB Large Intestine & 0.89 & 11.48 & 4.14 \\
\hline
\end{tabular}

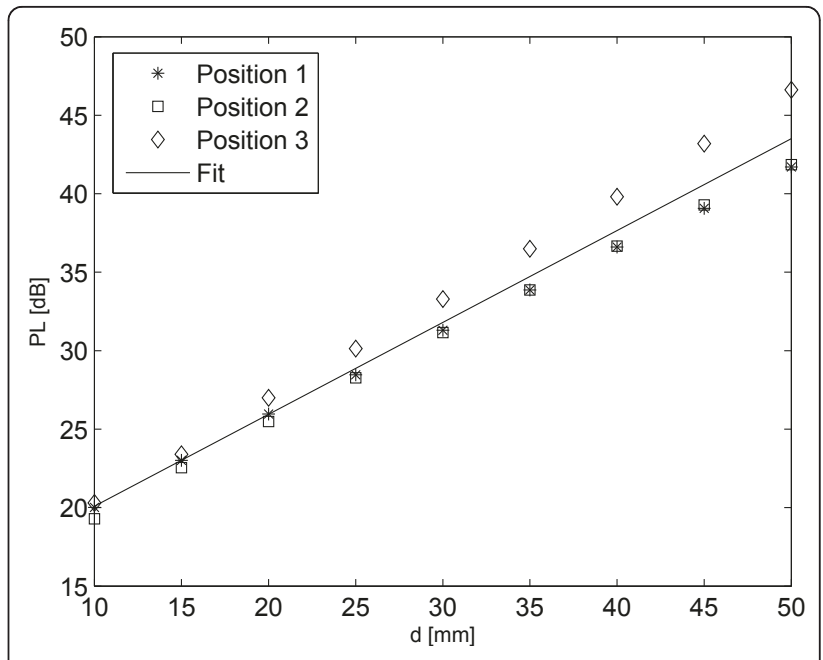

Figure 6 Path loss and the fitted model in esophagus.

distributions by plotting their quantiles against each other. The points on the graph lay close to the straight line suggesting that the data is normally distributed.

\section{3) Heterogeneous model for stomach-scenario II}

The PL of the simulated results for the three positions in the stomach versus the distances and their fit is shown in the Figure 8. At this position the Tx is placed in the stomach (Figure 2) and the Rx is separated up to a distance of $8 \mathrm{~cm}$. As the Rx moves from the stomach of the VFB to the liver a slight change is observed in the PL due to changes in the dielectric properties of the tissues (between 2 and $3 \mathrm{~cm}$ (Figure 8)).

The PL is modeled as follows:

$$
\left.\mathrm{PL}\right|_{\mathrm{dB}}=\left(10 \log _{10} e^{2}\right) \alpha_{3} d+\left.C_{3}\right|_{\mathrm{dB}}+\left.\chi_{3}\right|_{\mathrm{dB}},
$$

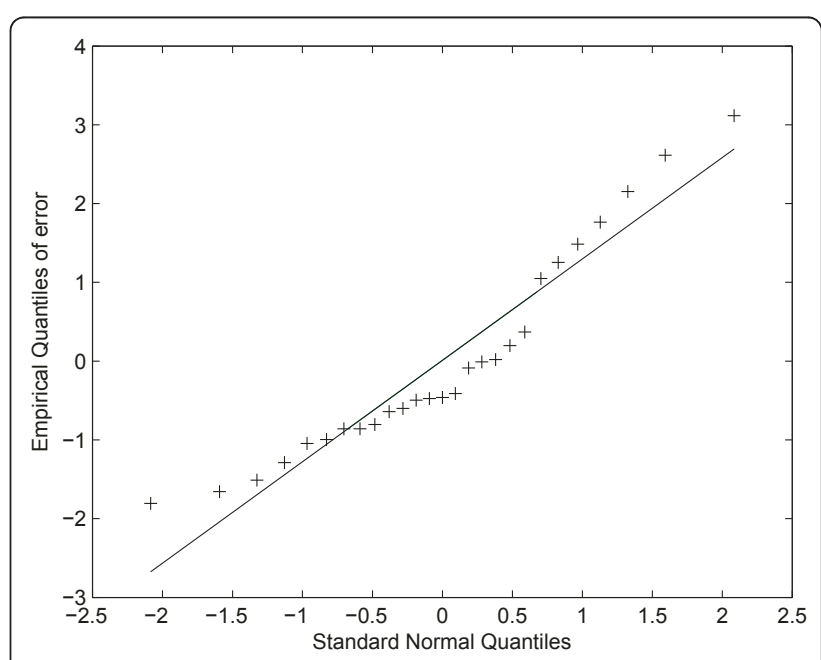

Figure $7 \mathrm{Q}-\mathrm{Q}$ plot of the empirical quantiles of error between the simulated PL and the PL model versus the standard normal quantiles in the esophagus. 


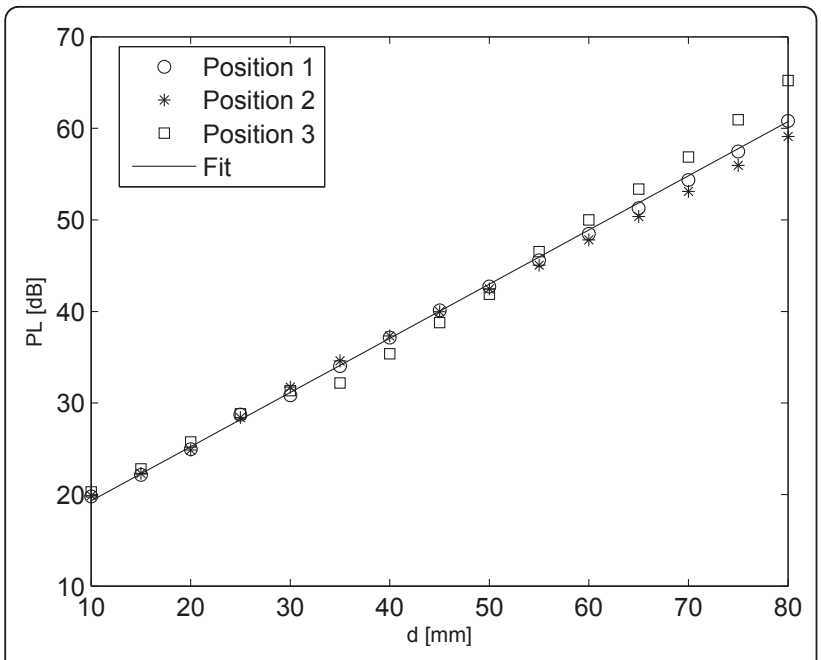

Figure 8 Path loss and the fitted model in stomach

where the parameter $\alpha_{3}$ is the effective attenuation constant $\left[\frac{1}{\mathrm{~cm}}\right],\left.C_{3}\right|_{\mathrm{dB}}$ is a constant and their values are listed in Table 2. $\left.\chi_{3}\right|_{\mathrm{dB}}$ follows a zero mean normal distribution with a SD of $1.22 \mathrm{~dB}$. Figure 9 shows the Q-Q plot of the empirical quantiles of the error between the PL model and the simulated PL results on the vertical axis to a theoretical standard normal distribution on the horizontal axis in the stomach of the VFB. It can be seen from the figure that they are in good agreement.

4) Heterogeneous model for small intestine-scenario III

In this section the simulated results are used to develop a PL model as a function of distance for the Tx placed at the intestine of the VFB at $2.45 \mathrm{GHz}$. In this scenario the $\mathrm{Rx}$ antenna traverses through various tissues as mentioned in Section III-A. The PL in the small

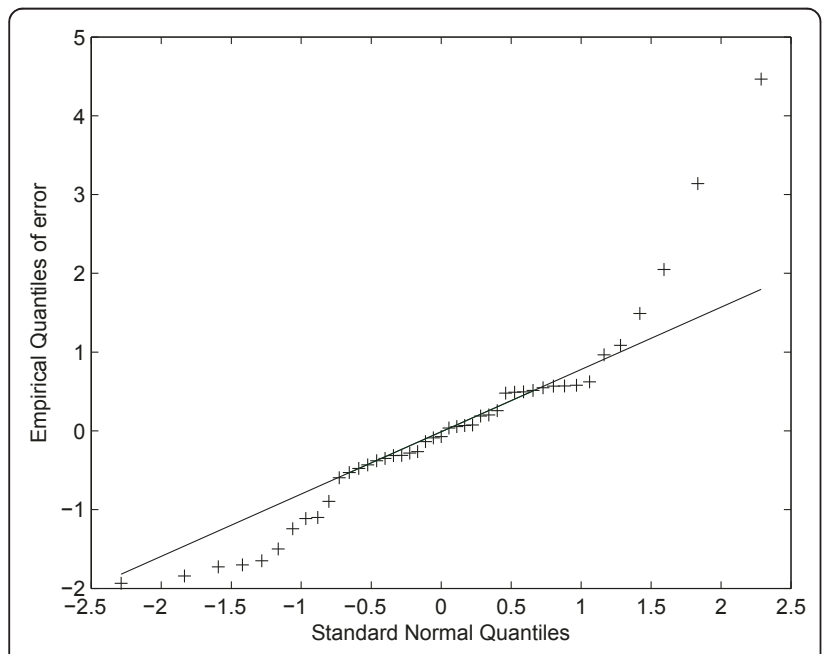

Figure $9 \mathrm{Q}-\mathrm{Q}$ plot of the empirical quantiles of error between the simulated PL and the PL model versus the standard normal quantiles in the stomach. intestine at three different positions as a function of distance and the fitted model is shown in Figure 10. The PL is modeled as follows:

$$
\left.\mathrm{PL}\right|_{\mathrm{dB}}=\left(10 \log _{10} e^{2}\right) \alpha_{4} d+\left.C_{4}\right|_{\mathrm{dB}}+\left.\chi_{4}\right|_{\mathrm{dB}},
$$

where the parameter $\alpha_{4}$ is the effective attenuation constant $\left[\frac{1}{\mathrm{~cm}}\right],\left.C_{4}\right|_{\mathrm{dB}}$ is a constant and their values are listed in Table 2. $\left.\chi_{4}\right|_{\mathrm{dB}}$ follows a zero mean normal distribution with a SD of $2.25 \mathrm{~dB}$. Figure 11 shows the Q$\mathrm{Q}$ plot of the empirical quantiles of the error between the PL model and the simulated PL results on the vertical axis to a theoretical standard normal distribution on the horizontal axis. Figure 11 shows that the empirical distribution agrees very well with the normal distribution.

\section{5) Heterogeneous model for large intestine-scenario IV}

Here the PL is modeled for the three positions of the Tx in the large intestine as shown in Figure 12 and it can be observed that the PL is high for a separation of 4 and $5 \mathrm{~cm}$ from the Tx antenna. At these distance the $\mathrm{Rx}$ antenna moves out of the large intestine into region of fat, thus increasing the PL. The PL is modeled as follows:

$$
\left.\mathrm{PL}\right|_{\mathrm{dB}}=\left(10 \log _{10} e^{2}\right) \alpha_{5} d+\left.C_{5}\right|_{\mathrm{dB}}+\left.\chi_{5}\right|_{\mathrm{dB}}
$$

where the parameters $\alpha_{5}$ is the effective attenuation constant $\left[\frac{1}{\mathrm{~cm}}\right],\left.C_{5}\right|_{\mathrm{dB}}$ is a constant and their values are listed in Table 2. $\left.\chi_{5}\right|_{\mathrm{dB}}$ follows a zero mean normal distribution with a SD of $4.14 \mathrm{~dB}$. This SD is larger than other scenarios as the antenna traverses through the large intestine, the fat and the dielectric properties of the two have vast differences. Figure 13 shows the Q-Q plot of the empirical quantiles of the error between the PL model and the simulated PL results on the vertical

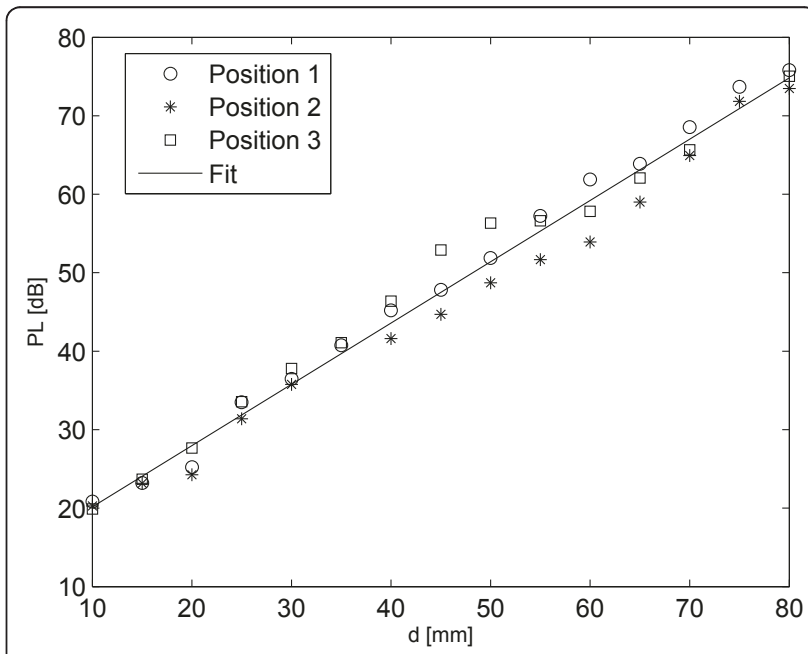

Figure 10 Path loss and the fitted model in small intestine. 


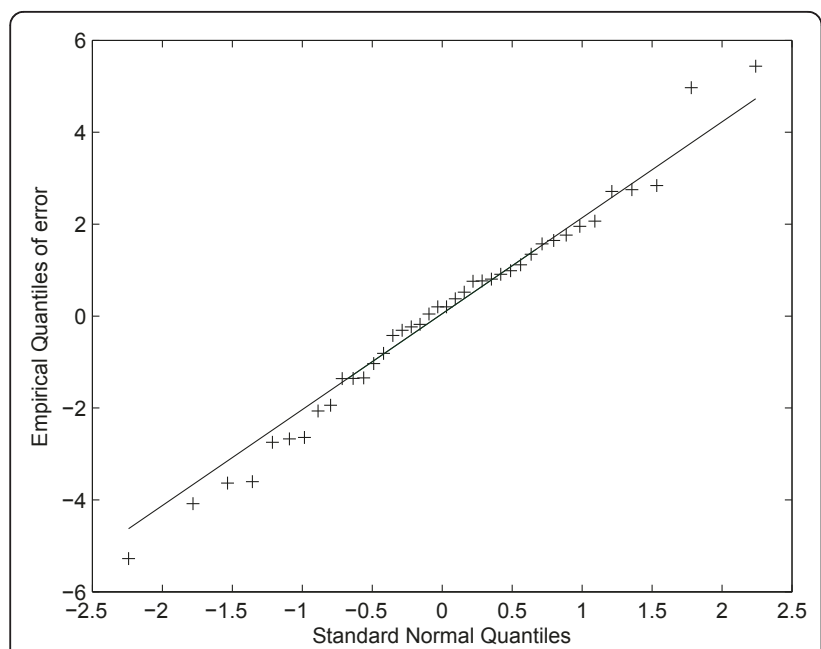

Figure $11 \mathrm{Q}-\mathrm{Q}$ plot of the empirical quantiles of error between the simulated PL and the PL model versus the standard normal quantiles in the small intestine.

axis to a theoretical standard normal distribution on the horizontal axis. It demonstrates that the empirical distribution agrees very well with the normal distribution.

\section{Discussion}

Figure 14 shows the PL in the homogeneous medium, the 95th percentile of the PL in heterogeneous VFB and the PL samples from all the scenarios. The 95th percentile is the value below which 95 percent of the PL values may be found. We use the 95th percentile as a safety margin to account for the error term which is denoted as $\left.\chi\right|_{\mathrm{dB}}$ in the PL model. From Figure 14 it can be seen that the 95th percentile of the PL in heterogeneous VFB lies above the PL in the homogeneous medium. Thus, if we were to design an in-body WBAN with $95 \%$

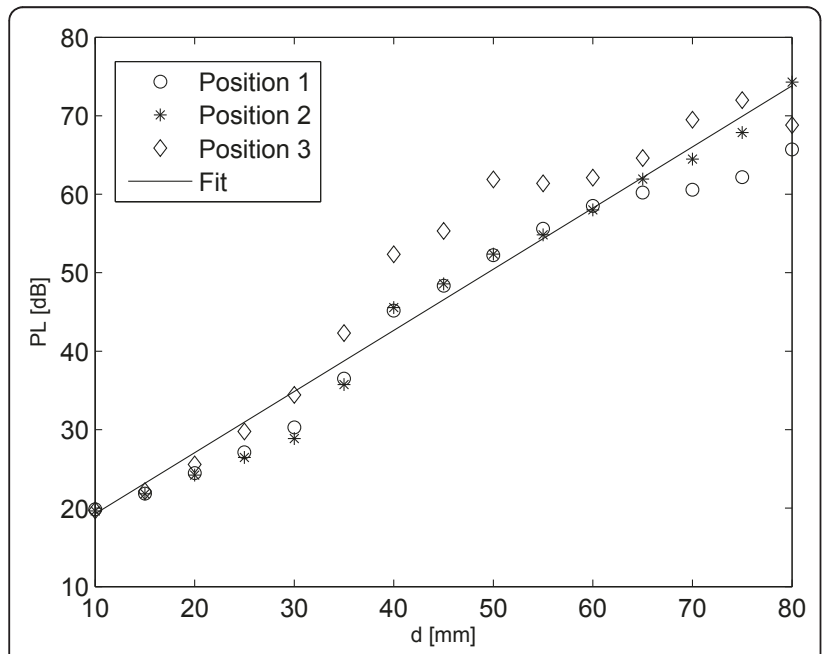

Figure 12 Path loss and the fitted model in large intestine.

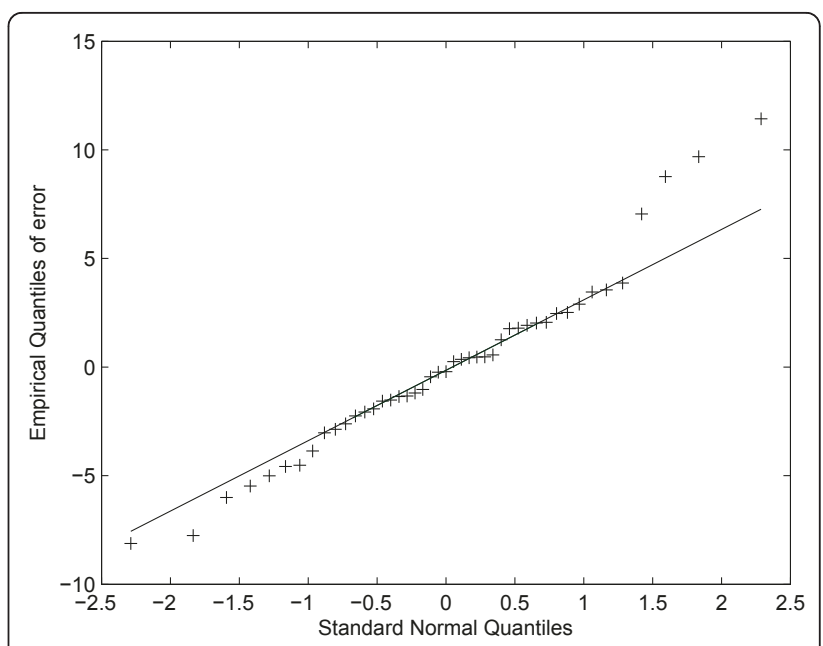

Figure $13 \mathrm{Q}-\mathrm{Q}$ plot of the empirical quantiles of error between the simulated PL and the PL model versus the standard normal quantiles in the large intestine.

coverage, link budget calculations will be more conservative than for the homogeneous medium and thus it is very important to perform the study of in-body WBAN using heterogeneous models. Since it is difficult to carry out measurements using heterogeneous medium, homogeneous medium can still be used for validation and equipment testing. However simulations using heterogeneous models will provide more conservative models for PL.

Table 2 lists all the attenuation constants $\alpha_{i}$, the constant $\left.C\right|_{\mathrm{dB}}$ and the SD from all the scenarios considered. It can be seen for the two scenarios of small intestine and large intestine the effective attenuation constants are higher than the attenuation constant of the homogeneous muscle tissue. The conductivity, $\sigma=3.17 \mathrm{~S} / \mathrm{m}$ of

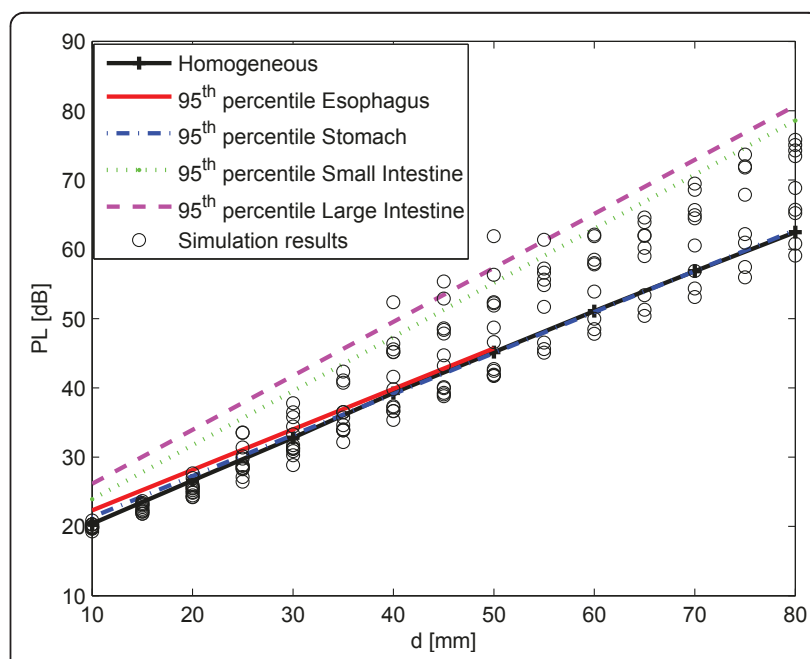

Figure 14 Path loss of the implantable antenna in the homogeneous medium and in heterogeneous VFB. 
the small intestine is much higher than the conductivity, $\sigma=2.01 \mathrm{~S} / \mathrm{m}$, of the muscle tissue causing more attentuation in the small intestine. In this scenario the $\mathrm{Rx}$ antenna traverses through various tissues such as the kidney $\left(\varepsilon_{\mathrm{r}}=52.74\right.$ and $\left.\sigma=2.43 \mathrm{~S} / \mathrm{m}\right)$, gall bladder $\left(\varepsilon_{\mathrm{r}}=\right.$ 68.36 and $\sigma=2.8 \mathrm{~S} / \mathrm{m})$, liver $\left(\varepsilon_{\mathrm{r}}=54.81\right.$ and $\sigma=2.25$ $\mathrm{S} / \mathrm{m})$, and also the artery $\left(\varepsilon_{\mathrm{r}}=58.26\right.$ and $\left.\sigma=2.54 \mathrm{~S} / \mathrm{m}\right)$. The tissues through which the antenna passes through have the conductivity higher as compared to the muscle tissue. In the large intestine the huge variation in the dielectric properties of the tissues (large intestine and the fat layer) through which the Rx traverses gives rise to higher attenuations. In case of the esophagus and the stomach, Table 2 shows that the effective attenuation constant lower than the muscle tissue medium as in both the scenario the Rx antenna traverses through tissues having lower conductivities compared to the muscle tissue. In case of the esophagus the tissues are esophagus $\left(\varepsilon_{\mathrm{r}}=62.15\right.$ and $\left.\sigma=2.2 \mathrm{~S} / \mathrm{m}\right)$ and lungs $\left(\varepsilon_{\mathrm{r}}=\right.$ 34.42 and $\sigma=1.24 \mathrm{~S} / \mathrm{m})$. In case of stomach the antenna traverses through the stomach lumen $\left(\left(\varepsilon_{\mathrm{r}}=\right.\right.$ 52.72 and $\sigma=1.74 \mathrm{~S} / \mathrm{m}$ ).

\section{Conclusions}

The path loss in homogeneous human muscle tissue and various heterogeneous media using implantable slot dipole conformal flexible antennas is investigated at 2.45 GHz. An in-body path loss model for the homogeneous medium and a heterogeneous human model is derived. Simulations based on FDTD and the fitted models show excellent agreement. It is observed from the considered scenarios, that the 95th percentile of the PL in heterogeneous VFB lies above the PL in the homogeneous medium. Thus, PL model in the heterogeneous VFB will help in understanding the power requirements for implants working at $2.45 \mathrm{GHz}$. The PL model in the heterogeneous human model for the considered deep tissue implant scenarios can also be used to evaluate the performance of in-body WBAN systems using well specified setups and to carry out link budget calculations.

\footnotetext{
Abbreviations

CPW: coplanar waveguide; ERC: European Radiocommunications Committee; FDTD: finite-difference time-domain; ICDs: implantable cardioverter defibrillators; ISM: industrial: scientific: and medical; MRI: magnetic resonance images; PL: path loss; PDMS: polydimethylsiloxane; Rx: receiver; SRD: short range device; SD: standard deviation; Tx: transmitter; WBAN: wireless body area network
}

\section{Author details}

'Department of Information Technology, WiCa, Ghent University/IBBT, Gaston Crommenlaan 8 Box 201, 9050 Ghent, Belgium ²Department of Information Technology, Electromagnetics Group, Ghent University, SintPietersnieuwstraat 41, 9000 Gent, Belgium ${ }^{3}$ Ghent University, ELINTEC-TFCG Technologiepark 914, 9052 Gent, Belgium

\section{Competing interests}

The authors declare that they have no competing interests

Received: 18 October 2010 Accepted: 3 August 2011

Published: 3 August 2011

\section{References}

1. B Latre, G Vermeeren, I Moerman, L Martens, SDF Louagie, P Demeester, Networking and propagation issues in body area networks. IEEE 11th Symposium on Communications and Vehicular Technology in the Benelux 2004, SCVT 2004, November 2004 (CD-ROM)

2. A Alomainy, $\mathrm{Y}$ Hao, Modeling and characterization of biotelemetric radio channel from ingested implants considering organ contents. Antennas and Propagation, IEEE Transactions. 57, 999-1005 (April 2009)

3. SKS Gupta, Y Prakash, E Elsharawy, L Schwiebert, Towards a propagation model for wireless biomedical applications. IEEE International Conference on Communications. 3, 1993-1997 (May 2003)

4. A Johansson, Wireless communication with medical implants (Ph.D. dissertation, Lund University, 2004)

5. K Ito, Wei Xia, Masaharu Takahashi, K Saito, An Implanted Cavity Slot Antenna for Medical Communication Systems, in 3rd European Conference on Antennas and Propagation (EUCAP), Berlin, Germany. 718-721 (March 2009)

6. D Kurup, W Joseph, G Vermeeren, L Martens, Path loss model for in-body communication in homogeneous human muscle tissue. IET Electronics Letters, 453-454 (April 2009)

7. M Scarpello, D Kurup, H Rogier, D Vande Ginste, F Axisa, J Vanfleteren, W Joseph, L Martens, G Vermeeren, Design of an implantable slot dipole conformal flexible antenna for biomedical applications. Submitted to IEEE Transactions on Antennas and Propagation (2010)

8. B Barth, K Donovan, V Fox, Endoscopic placement of the capsule endoscope in children. Gastrointestinal Endoscopy. 60, 818-821 (November 2004). doi:10.1016/S0016-5107(04)02052-8

9. E Seidman, M Dirks, Capsule endoscopy in the pediatric patient. Current Treatment Options Gastroenterol. 9, 416-22 (September 2006). doi:10.1007/ BF02738531

10. H Kavin, J Berman, T Martin, A Feldman, K Forsey-Koukol, Capsule endoscopy for a 2.5-year-old child: Obscure gastrointestinal bleeding from mixed, juvenile, capillary hemangioma-angiomatosis of the jejunum. Pediatrics. 117, 539-543 (February 2006). doi:10.1542/peds.2005-0710

11. M Thomson, A Fritscher-Ravens, S Hall, N Afzal, P Ashwood, C Swain, Endoluminal gastroplication in children with significant gastro-oesophageal reflux disease. Gut. 53, 1745-50 (April 2004). doi:10.1136/gut.2004.041921

12. FCC OET Bulletin 65, Revised Supplement C, Evaluating Compliance with FCC Guidelines for Human Exposure to Radiofrequency Electromagnetic Fields. Federal Communication Commission, Office of Engineering and Technology (June 2001)

13. European Radiocommunications Committee, ERC recommendations 70-03 relating to the use of short range devices (SRD), in Eur. Postal Telecommunications Administration Conf., CEPT-ERC 70-03, Annex 12, Tromso, Norway, (1997)

14. C Gabriel, S Gabriel, Compilation of the dielectric properties of body tissues at RF and microwave frequencies. Tech. Rep. AL/OE-RE-1996-0037. http:// niremf.ifac.cnr.it/docs/DIELECTRIC/home.html (1996)

15. SPEAG-Schmid and Partner Engineering AG, Reference manual for the semcad simulation platform for electromagnetic compatibility, antenna design and dosimetry, (2001)

16. SJ Orfanidis, Electromagnetic Waves and Antennas, http://www.ece.rutgers. edu/ orfanidi/ewa/ (2008)

doi:10.1186/1687-1499-2011-51

Cite this article as: Kurup et al.: In-body path loss models for implants in heterogeneous human tissues using implantable slot dipole conformal flexible antennas. EURASIP Journal on Wireless Communications and Networking 2011 2011:51 